\title{
VARIABILIDADE DA FREQUÊNCIA CARDÍACA EM DIFERENTES POSIÇÕES CORPORAIS E DURANTE MASR EM AMPUTADO UNILATERAL DE MEMBRO INFERIOR
}

\section{Andréa Lúcia Gonçalves da Silva}

Vice-Coordenadora do Curso de Fisioterapia e Docente do Departamento de Educação Física e Saúde da Universidade de Santa Cruz do Sul (UNISC), Brasil.

\section{Ana Paula Dattein Peiter}

Fisioterapeuta formada pelo curso de Fisioterapia da Universidade de Santa Cruz do Sul (UNISC), Brasil.

\section{Cássia da Luz Goulart}

Doutoranda em Fisioterapia Cardiopulmonar na Universidade Federal de São Carlos (UFSCar), Brasil.

\section{Paloma Borba Schneiders}

Bolsista de Iniciação Científica pela Universidade de Santa Cruz do Sul (UNISC), Curso de Fisioterapia (UNISC), Santa Cruz do Sul (RS), Brasil.

\section{Elisabete Antunes San Martin}

Bolsista de Iniciação Científica pela Universidade de Santa Cruz do Sul (UNISC), Curso de Fisioterapia (UNISC), Santa Cruz do Sul (RS), Brasil.

\section{Renata Trimer}

Docente do Departamento de Educação Física e Saúde da Universidade de Santa Cruz do Sul (UNISC), Brasil.

\section{Angela Cristina Ferreira da Silva}

Coordenadora do Departamento de Educação Física e Saúde da Universidade de Santa Cruz do Sul (UNISC), Brasil.
RESUMO: A amputação leva a redução da mobilidade e atividade física afetando os sistemas musculoesquelético, respiratório e cardiovascular. Nosso objetivo foi analisar a variabilidade da frequência cardíaca (VFC) em diferentes posições corporais e durante a Manobra de Arritmia Sinusal Respiratória (MASR) em amputados unilaterais de membros inferiores (MMII) protetizados. Estudo transversal, amostra de conveniência, avaliou 07 amputados homens de origem traumática e registradas as variáveis clínicas e VFC nas posições supino, ortostase, sedestação (10 minutos) e durante a MASR (4 minutos). Em repouso os sujeitos apresentam predominância da modulação simpática que se acentua quando assume a posição de ortostase $(\mathrm{p}=<0.01)$ e atenuação da complexidade autonômica $(\mathrm{p}=0,04)$. Os amputados não responderam de modo adequado à MASR e observou-se alteração na complexidade da VFC $(0,04)$. Amputados unilaterais de MMII protetizados demonstraram comportamento esperado durante a mudança ativa de postura e respostas adversas à MASR.

PALAVRAS-CHAVE: Amputação; Arritmia Sinusal; Frequência Cardíaca; Sistema Nervoso Autônomo.

\section{VARIABILITY OF HEART BEAT FREQUENCY IN DIFFER- ENT BODY POSITIONS AND DURING MASR IN A PER- SON WITH LOWER MEMBER UNILATERAL AMPUTATION}

ABSTRACT: Amputation causes decrease of mobility and physical activities, affecting the muscle-skeleton, respiratory and cardiovascular systems. Variability of heart frequency (VFC) at different body positions and during Respiratory Sinus Arrhythmia (RSA) was analyzed in people with amputation of unilateral lower members with prosthesis (MMII). Current transversal study, with convenience sample, evaluated seven males with trauma-caused amputations. Clinical variations and VFC were registered with regard to supine position, orthostasis, sedestation (10 $\mathrm{min}$ ) and during RSA (4 min). The subjects at rest showed a predominance in increasing sympathetic modulation as the orthostatic position is achieved ( $\mathrm{p}=<0.01)$ and attenuation of autonomic complexity $(p=0.04)$. People with amputation failed to respond adequately to RSA and alterations were detected in VFC complex (0.04). Unilateral amputated people with prosthesis showed expected behavior during active changes in posture and adverse responses to RSA.

KEY WORDS: Amputation; Sinus arrhythmia; Heart beating frequency; Autonomous nervous system. 


\section{INTRODUÇÃO}

A amputação de membro inferior (MI) resulta na redução da mobilidade e da atividade física dos sujeitos, afetando nos sistemas musculoesquelético, respiratório e cardiovascular ${ }^{1}$. E as alterações no estilo de vida dos amputados, o estresse físico e mental, assim como o estresse oxidativo, podem desencadear mecanismos de anormalidades do miocárdio e do tecido de condução cardíaco, o que predispõem ao desequilíbrio simpático-vagal ${ }^{2-4}$.

Um dos principais instrumentos de avaliação do Sistema Nervoso Autônomo (SNA) é a análise da Variabilidade da Frequência Cardíaca (VFC) que consiste em um método não invasivo, que descreve as flutuações nos intervalos R-R dos batimentos cardíacos ${ }^{5}$. A análise da VFC reflete a interação dos sistemas simpático e parassimpático ao sistema cardiovascular, através de terminações aferentes e eferentes no miocárdio, nodo sinusal e nodo atrioventricular ${ }^{6}$. Esta interação é de suma importância no aumento ou redução do desempenho cardíaco em diferentes circunstâncias ${ }^{7}$, sendo estes influenciados pelos barorreceptores, quimiorreceptores, receptores atriais e ventriculares, além de modificações no sistema respiratório, vasomotor e termorregulador ${ }^{8}$. No entanto, as respostas do controle autonômico frente às modificações do posicionamento do corpo no espaço e durante a Manobra de Arritmia Sinusal Respiratória (MASR), particularmente em amputados de membros inferiores, precisam ser esclarecidas.

Peles et al. ${ }^{3}$ observaram a resposta no domínio da frequência da análise da VFC em supinação e ortostase de amputados traumáticos, em que encontraram um predomínio da modulação parassimpática em ortostase. Já Cachadiña et al. ${ }^{9}$ avaliaram a VFC em homens amputados com dor fantasma, concluindo que a disfunção autonômica também parece estar envolvida nas alterações cardiovasculares do amputado como reflexo de uma atividade simpática anormal do membro residual. Contudo, nestes estudos, não foram avaliados os índices autonômicos cardíacos durante o repouso em supinação, sedestação, ortostase e na MASR de homens amputados traumáticos. Assim, estudos neste contexto são necessários como forma de caracterizar quais índices de VFC poderiam explicar melhor as alterações na modulação autonômica cardíaca de amputados de membro inferior.
Desta forma, nosso objetivo foi analisar os índices de VFC de homens amputados traumáticos unilaterais em diferentes posições corporais e durante a MASR. Nós hipotetizamos que amputados apresentem respostas prejudicadas no controle autonômico cardíaco frente a mudanças posturais e durante a MASR.

\section{METODOLOGIA}

Estudo de delineamento transversal, com amostragem de conveniência, que avaliou homens com amputação traumática unilateral de membro inferior e protetizados, junto ao Serviço de Reabilitação Física da Universidade de Santa Cruz do Sul (UNISC), no município de Santa Cruz do Sul (RS), Brasil. O protocolo do estudo foi conduzido de acordo com a declaração de Helsinki e foi aprovado pelo Comitê de Ética em Pesquisa da Universidade de Santa Cruz do Sul através do protocolo número 1.361.054 e todos os sujeitos do estudo assinaram o Termo de Consentimento Livre e Esclarecido.

\section{SUJEITOS}

Foram incluídos nesta pesquisa homens amputados traumáticos unilaterais de membro inferior protetizados, independente do nível e do tempo de amputação, idade superior a 18 anos, com boa cognição e que aceitaram voluntariamente participar desta pesquisa. Foram excluídos da pesquisa amputados de etiologia diabética, com doença arterial vascular, com déficit neurológico ou ortopédico, amputados não protetizados, bem como portadores de cardiopatia/arritmia ou doença metabólica não controlada, tais como alterações na tireóide e hipertensão arterial sistêmica.

\section{MENSURAÇÕES}

A coleta de dados ocorreu em sala reservada, silenciosa e climatizada a $22{ }^{\circ} \mathrm{C}$. Os sujeitos da pesquisa foram orientados a evitar a ingesta de alimentos e bebidas estimulantes nas 24 horas antecedentes à avaliação, assim como não fumar ou realizar exercícios extenuantes. Foram coletados os dados clínicos dos pacientes através de 
questionário padrão onde constavam sexo, idade, causa, nível, data da amputação, tempo de utilização da prótese e medicamentos utilizados. Consumo estimado de oxigênio $\left(\mathrm{VO}_{2}\right)$ foi obtido através do Duke Activity Status Index (DASI) e classificado conforme Cooper (1982) ${ }^{10}$. Além disso, foram registradas medidas antropométricas como peso ${ }^{11,12}$, altura, calculado o IMC, circunferência abdominal, circunferência da cintura e do quadril, bem como a Relação Cintura-Quadril (RCQ).

\section{ANÁLISE DA VARIABILIDADE DA FREQUÊNCIA CARDÍA-} CA

O registro da Frequência Cardíaca (FC) foi realizado através do cardiofrequencímetro (sistema Polar ${ }^{\circledR}$ ), onde uma cinta elástica foi posicionada ao redor do tronco de cada sujeito ao nível do terço inferior do esterno. Os sinais foram continuamente transmitidos para a unidade receptora através de campo eletromagnético (relógio colocado no pulso direito), sendo todos os dados transferidos posteriormente para um computador utilizando o software Pro-Trainer 5 TM ${ }^{\circledR}$ Polar.

O intervalo RR (iRR) foi registrado utilizando o sistema Polar@ em repouso, seguindo a ordem: posição supina (10 minutos), ortostase (10 minutos), sedestação (10 minutos) e MASR (4 minutos, em sedestação). Nessa manobra, os pesquisadores instruíram os sujeitos a realizar uma sequência de inspirações e expirações profundas e lentas. Cada ciclo respiratório durou 10 segundos (5 segundos de inspiração, 5 segundos de expiração). Os voluntários seguiram os comandos verbais dos pesquisadores para manter a frequência respiratória de seis respirações por minuto, o que se espera induzir ao máximo de arritmia sinusal respiratória ${ }^{13}$.

Para a análise da VFC foi utilizado software $\mathrm{Ku}$ bios $H R V \otimes$ versão 2.1. Foi examinado trechos de iRR e selecionado o segmento mais estável contendo 256 intervalos. A média dos intervalos da FC (iRR), desvio padrão do iRR em milissegundos (desvio padrão do iRR), média da frequência cardíaca (Média FC), desvio padrão da frequência cardíaca (desvio padrão da $\mathrm{FC}$ ), raiz quadrada da média da soma dos quadrados das diferenças entre os intervalos RR (rMSSD) e o índice triangular (RRtri indice) foram obtidos para análise no domínio do tempo.
Para análise do domínio da frequência, foram obtidos os índices de Baixa frequência (BF), alta frequência (AF) e a relação BF/AF (equilíbrio simpato-vagal). A análise da VFC não-linear deu-se a partir dos índices de Poincare desvio padrão da medida de dispersão de pontos perpendiculares a linha de identidade (SD1), desvio padrão da medida de dispersão de pontos ao longo da linha de identidade (SD2), Aproximate Entropy (ApEn), Sample Entropy e (SampEn).

\section{ANÁLISE ESTATÍSTICA}

O cálculo do tamanho da amostra foi realizado a priori, utilizando o software Gpower (3.1.9.2), para atingir um poder estatístico de $80 \%(\beta=0,20)$, com $\alpha=0,05$, calculou-se que um mínimo de 11 pacientes amputados são necessários. Destacamos que nosso $\mathrm{N}$ amostral total é $\mathrm{N}=7$, porém avaliamos 14 pacientes amputados, mas destes 7 possuem diabetes tipo II, fator de exclusão do estudo.

Os dados foram analisados utilizando o programa estatístico Sigmaplot ${ }^{\circledR}$ (versão 11.0, Systat Software Inc., San José, CA, EUA) e posteriormente expressos quanto à normalidade pelo teste de Shapiro-Wilk e apresentados descritivamente como média e desvio padrão (paramétrico) ou como mediana e intervalo mínimo e máximo (não paramétrico). Para rejeitar a hipótese nula, utilizou-se o teste $\mathrm{t}$ de Student para dados paramétricos e o teste de Mann-Wbitney para dados não paramétricos. Residuais foram avaliados sob os pressupostos de normalidade, variância constante e independência. Foi considerado significativo $\mathrm{p}<0,05$.

\section{RESULTADOS}

Um total de 7 homens amputados traumáticos unilaterais de membro inferior foram avaliados a partir dos critérios de inclusão. As características clínicas e antropométricas estão descritas na Tabela 1, sendo predominantemente composto por sujeitos em idade adulto jovem, em sua maioria com peso ideal, diferentes classificações de risco cardiovascular, amputação transfemural e aptidão física muito fraca com base no $\mathrm{VO}_{2}$ estimado através da classificação de Cooper (1982) ${ }^{10}$. 
Tabela 1. Características clínicas dos sujeitos amputados traumáticos unilateral de membro inferior

\begin{tabular}{|c|c|}
\hline Variáveis & Amputados $(n=7)$ \\
\hline Idade (anos) & $30.8 \pm 8.8$ \\
\hline Sexo Masculino, n (\%) & $7(100.0)$ \\
\hline \multicolumn{2}{|l|}{ Nível Amputação, n (\%) } \\
\hline Transtibial & $3(42.8)$ \\
\hline Transfemural & $4(57.2)$ \\
\hline $\mathrm{IMC}\left(\mathrm{Kg} / \mathrm{m}^{2}\right)$ & $27.6 \pm 6.2$ \\
\hline \multicolumn{2}{|l|}{ Classificação IMC, n (\%) } \\
\hline Normal & $4(57.1)$ \\
\hline Sobrepeso & $2(28.6)$ \\
\hline Obesidade & $1(14.3)$ \\
\hline RCQ & $0,85 \pm 0$ \\
\hline \multicolumn{2}{|l|}{ Classificação RCQ, n (\%) } \\
\hline Baixo & $2(28,6)$ \\
\hline Moderado & $2(28,6)$ \\
\hline Alto & $2(28,6)$ \\
\hline Muito Alto & $1(14,3)$ \\
\hline Circunferência Abdominal (cm) & $98,3 \pm 10,4$ \\
\hline \multicolumn{2}{|l|}{ DASI } \\
\hline Escore Total (pontos) & $36.8 \pm 6.6$ \\
\hline $\mathrm{VO}_{2}$ máx $\left(\mathrm{ml} \cdot \mathrm{kg}^{-1} \cdot \mathrm{min}^{-1}\right)$ & $25.4 \pm 2.8$ \\
\hline \multicolumn{2}{|l|}{ Classificação $\mathrm{VO}_{2}, \mathrm{n}(\%)$} \\
\hline Muito fraco & $7(100.0)$ \\
\hline
\end{tabular}

Dados expressos em frequência, média, desvio padrão; n: número amostral; IMC: Índice de massa corporal; RCQ: Relação Cintura-Quadril; DASI: Duke Activity Status Index; $\mathrm{VO}_{2}$ : Consumo de oxigênio.

Quando analisados os trechos mais estáveis da VFC, tanto no repouso em posição supino quanto em sedestação, encontramos um predomínio nas bandas de BF e BF/AF e redução do AF (Tabela 2). Quando os sujeitos assumem a posição ortostase, observamos uma acentuação nas bandas $\mathrm{BF}(\mathrm{p}<0,01), \mathrm{BF} / \mathrm{AF}(\mathrm{p}<0,01)$ e redução da complexidade do SNA (ApEn p < 0,01), indicando um aumento da modulação simpática e atenuação parassimpática durante a passagem da posição de supino para ortostase. Nos domínios não-lineares verificou-se a redução do SampEn $(\mathrm{p}=0.01)$, indicando uma redução da complexidade da VFC com a mudança ativa de postura para ortostase (Tabela $2)$.

Tabela 2. Índices de VFC dos amputados traumáticos unilateral de membro inferior

(Continua)

\begin{tabular}{|c|c|c|c|c|c|}
\hline \multicolumn{6}{|c|}{ Sujeitos Amputados $(n=7)$} \\
\hline Variáveis & SUPINO & IC (95\%) & ORTOSTASE & IC (95\%) & $p$ \\
\hline Média iRR (ms) & $817.0 \pm 152.6$ & $817(675-958)$ & $686.3 \pm 109.2$ & $686(778-843)$ & 0.09 \\
\hline DP iRR (ms) & $28.9 \pm 17.9$ & $28(12-45)$ & $23.0 \pm 16.1$ & $36(23-49)$ & 0.53 \\
\hline Média FC (1/min) & $75.7 \pm 13.5$ & $75(63-88)$ & $89.3 \pm 12.7$ & $70(63-77)$ & 0.07 \\
\hline $\mathrm{DP} F C(1 / \mathrm{min})$ & $2.7 \pm 1.2$ & $2(1-3)$ & $3.2 \pm 1.6$ & $3(2-4)$ & 0.60 \\
\hline rMSSD (ms) & $26.0 \pm 20.7$ & $26(6-45)$ & $13.1 \pm 9.6$ & $28(13-44)$ & 0.16 \\
\hline RRtri índice & $7.4 \pm 3.6$ & $7(4-10)$ & $6.4 \pm 4.1$ & $9(5-12)$ & 0.64 \\
\hline $\mathrm{BF}(\mathrm{nu})$ & $67.3 \pm 14.4$ & $67(53-80)$ & $85.5 \pm 3.1$ & $71(50-92)$ & $<0.01$ \\
\hline
\end{tabular}


(Continua)

\begin{tabular}{|c|c|c|c|c|c|}
\hline \multicolumn{6}{|c|}{ Sujeitos Amputados $(n=7)$} \\
\hline Variáveis & SUPINO & IC (95\%) & ORTOSTASE & IC (95\%) & $p$ \\
\hline $\mathrm{AF}(\mathrm{nu})$ & $32.4 \pm 14.7$ & $32(18-46)$ & $14.4 \pm 3.1$ & $28(7-49)$ & $<0.01$ \\
\hline $\mathrm{BF} / \mathrm{AF}^{*}$ & $2.4(1.8-3.4)$ & $2(1-3.5)$ & $6.1(5.0-6.7)$ & $4(1-7)$ & $<0.01$ \\
\hline SD1* & $15.1(10.5-22.5)$ & $18(4-32)$ & $6.5(3.3-13.7)$ & $20(9-31)$ & 0.16 \\
\hline $\mathrm{SD} 2 *$ & $33.7(24.1-48.5)$ & $36(16-55)$ & $23.7(11.7-52.6)$ & $46(30-62)$ & 0.66 \\
\hline Aproximate Entropy & $1.2 \pm 0.0$ & $1(0.9-1)$ & $0.98 \pm 0.0$ & $0.9(0.91-1)$ & 0.32 \\
\hline Sample Entropy & $1.7 \pm 0.2$ & $1.7(1.5-1.9)$ & $1.4 \pm 0.2$ & $1.5(1.1-1.8)$ & 0.04 \\
\hline $\begin{array}{l}\text { Notes: Notas: Os dados } \\
\text { usado para dados paran } \\
\text { de Mann-Whitney. Abre } \\
\text { rMSSD: raiz quadrada d } \\
\text { de baixa frequência; AF: } \\
\text { de plotagem Poincare; } 1\end{array}$ & $\begin{array}{l}\text { como média } \pm \text { c } \\
\text { le Mann-Whitne } \\
\text { bilidade da freq } \\
\text { cças sucessivas a } \\
\text { lência; nu: unid } \\
\text { arritmia sinusal }\end{array}$ & $\begin{array}{l}\text { drão ou como } \\
\text { do para dados } \\
\text { ardíaca; iRR: in } \\
\text { do entre R-R ac } \\
\text { malizadas; BF/A } \\
\text { ória. }\end{array}$ & $\begin{array}{l}\text { na e (intervalo in } \\
\text { ramétricos. Níve } \\
\text { da FC; DP: desvi } \\
\text { es; RRtri índice: í } \\
\text { ção entre as banc }\end{array}$ & $\begin{array}{l}\text { dartil). O teste } \\
\text { significância: } \mathrm{P} \\
\text { drão; FC: frequ } \\
\text { e triangular de } \\
\text { F e AF; SD1 e S }\end{array}$ & $\begin{array}{l}\text { tudent f } \\
, 05 . \text { *tes } \\
\text { a cardíac } \\
\text { BF: band } \\
\text { arâmetrc }\end{array}$ \\
\hline
\end{tabular}

Durante a MASR, em comparação dos índices de VFC em sedestação, apesar de não significativo os sujeitos amputados curiosamente demonstraram um aumento da modulação simpática com aumento da banda de BF e da relação $\mathrm{BF} / \mathrm{AF}$ e redução do AF, resposta esta oposta à esperada na MASR. Adicionalmente, os índices ApEn ( $\mathrm{p}=0.03)$ e SampEn $(\mathrm{p}=0.04)$ reduziram significativamente, sugerindo piora na complexidade durante a MASR (Tabela 3 ).

Tabela 3. Índices de VFC dos amputados traumáticos unilateral de membro inferior

\begin{tabular}{lccccc|}
\hline \multicolumn{1}{c}{ Variáveis } & \multicolumn{5}{c}{ Sujeitos Amputados $(\mathbf{n}=7)$} \\
\hline Média iRR (ms) & SEDESTAÇÃO & IC (95\%) & MASR & IC (95\%) & p \\
DP iRR (ms) & $767.0 \pm 87.7$ & $767(685-848)$ & $710.4 \pm 121.0$ & $710(598-822)$ & 0.33 \\
Média FC (1/min) & $30.7(22.7-33.8)$ & $27(16-38)$ & $26.3(17.8-55.8)$ & $41(6-75)$ & 0.84 \\
DP FC (1/min) & $79.3 \pm 9.4$ & $79(70-88)$ & $86.8 \pm 12.3$ & $86(75-98)$ & 0.22 \\
rMSSD (ms) & $3.2 \pm 1.1$ & $3(2-4)$ & $5.0 \pm 4.2$ & $5(1-9)$ & 0.29 \\
RRtri índice & $21.9(13.4-27.8)$ & $19(11-28)$ & $19.6(10.3-32.7)$ & $28(1-54)$ & 0.90 \\
BF (nu) & $7.0(5.0-8.7)$ & $6(4-9)$ & $7.1(5.5-13.8)$ & $9(3-14)$ & 0.36 \\
AF (nu) & $74.6 \pm 9.9$ & $74(65-83)$ & $81.1 \pm 11.9$ & $81(70-92)$ & 0.29 \\
BF/AF* & $25.2 \pm 9.9$ & $25(16-34)$ & $18.8 \pm 11.9$ & $18(7-29)$ & 0.29 \\
SD1* & $2.6(2.3-5.7)$ & $3(1-5)$ & $4.7(3.7-7.3)$ & $6(1-12)$ & 0.25 \\
SD2* & $15.5(9.5-19.7)$ & $14(7-20)$ & $13.9(7.3-23.2)$ & $20(1-38)$ & 0.90 \\
Aproximate Entropy & $39.8(30.7-44.1)$ & $36(22-51)$ & $34.5(24.1-75.2)$ & $54(9-99)$ & 0.38 \\
Sample Entropy & $0.9 \pm 0.0$ & $0.99(0.93-1)$ & $0,8 \pm 0.1$ & $0.8(0.7-0.9)$ & 0.03 \\
\hline Notes: Notas: Os & $1.5 \pm 0.1$ & $1.5(1.3-1.6)$ & $1.1 \pm 0.3$ & $1(0.7-1.5)$ & 0.04 \\
\hline
\end{tabular}

Notes: Notas: Os dados são apresentados como média \pm desvio padrão ou como mediana e (intervalo interquartil). O teste $t$ de Student foi usado para dados paramétricos e o teste de Mann-Whitney foi usado para dados não paramétricos. Nível de significância: $\mathrm{P}<0,05$. *teste de Mann-Whitney. Abreviaturas: VFC: variabilidade da frequência cardíaca; iRR: intervalo da FC; DP: desvio padrão; FC: frequência cardíaca; rMSSD: raiz quadrada da média das diferenças sucessivas ao quadrado entre R-R adjacentes; RRtri índice: índice triangular de VFC; BF: banda de baixa frequência; AF: banda de alta frequência; nu: unidades normalizadas; BF/AF: relação entre as bandas BF e AF; SD1 e SD2: parâmetros de plotagem Poincare; MASR: manobra de arritmia sinusal respiratória. 


\section{DISCUSSÃO}

Até onde sabemos este é o primeiro estudo que avalia a mudança ativa de postura e a MASR em homens amputados de membro inferior e protetizados, de causa traumática. Os nossos principais achados foram: a) amputados unilaterais de membro inferior e protetizados apresentam uma predominância da modulação simpática em repouso; b) durante a mudança ativa de postura para ortostase estes sujeitos apresentam comportamento adequado da modulação autonômica com ativação da modulação simpática e da complexidade autonômica; c) na MASR os amputados apresentaram uma resposta oposta à esperada com ativação da modulação simpática e diminuição da complexidade autonômica.

Contraditoriamente aos resultados por nós encontrados, Cachadiña et al. ${ }^{9}$ avaliaram 35 amputados em repouso na posição supino, e verificaram que todos os sujeitos apresentaram uma redução nos valores de BF, indicando baixa modulação da atividade simpática. Entretanto, é importante ressaltar que eles avaliaram sujeitos em diferentes níveis de amputação com e sem presença de dor no membro residual.

Nós especulamos que amputados de membros inferiores de origem traumática passam por um trauma emocional e físico que o confrontam com uma perda irreparável, que afeta toda a sua vida ${ }^{14}$. A dor residual e somatizada, por possíveis alterações psicossomáticas, é capaz de alterar o SNA uma vez que ela é baseada na atividade simpática anormal do membro residual. Além disso, o sistema nervoso simpático e sistema hormonal adrenomedular respondem de forma extremamente sensível aos estressores emocionais de perda imediata ${ }^{3}$. Afora isto, é importante reconhecer que outros fatores, tais como a dieta (consumo de cafeína e álcool) e estresse físico e mental, podem influenciar as discrepâncias entre os estudos ${ }^{15}$. Desconhecemos publicação em sujeitos amputados que abordem tal temática porém em outras populações de doentes já foram observados este padrão.

Referente aos achados da mudança ativa de postura para ortostase, estes podem ocorrer devido à provocação do acúmulo de fluxo sanguíneo nos membros inferiores, promovido pela carga ortostática que, por sua vez, provoca aumento do estímulo simpático e elevação da pressão arterial ${ }^{7}$. Este comportamento foi verificado nos estudos de Jiang et al. ${ }^{16}$ e Mahananto et al..$^{17}$, realizados com sujeitos depressivos e saudáveis respectivamente, onde durante a mudança postural de sedestação para ortostase, houve uma maior ativação da modulação simpática e atenuação vagal. Este aumento da modulação autonômica simpática em amputados também pode ser atribuído à irritação mecânica do coto pela prótese ${ }^{18}$.

Pertinente aos valores não-lineares $\mathrm{da} \mathrm{VFC}$, o único estudo que avaliou a mudança ativa de posição em amputados transfemurais encontrou uma diminuição da complexidade global e eles sugeriram que a modulação vagal tem um comportamento mais caótico do que a simpática ${ }^{19}$, o que vem ao encontro dos nossos resultados onde na posição de repouso (supino) os valores de ApEn foram superiores ao encontrado na posição ortostase. Adicionalmente, Vuksanović e $\mathrm{Gal}^{20}$, ao investigarem as características lineares e não-lineares da VFC em 60 indivíduos saudáveis, verificaram que a entropia da modulação autonômica cardíaca está diretamente associada ao envelhecimento.

Durante a MASR, os sujeitos estudados apresentaram uma resposta oposta quanto aos índices da VFC esperados, verificados através de uma hiperatividade simpática, redução do tônus parassimpático e piora da complexidade autonômica. Sendo que a MASR é uma técnica que induz a estimulação do SNA, promovendo uma ativação do sistema parassimpático exclusivamente, alterando o padrão respiratório para induzir a distensão do nó sinusal, como resultado de mudanças no retorno venoso durante a respiração profunda ${ }^{2}$. Ademais, Fenley et al. ${ }^{21}$, no intuito de avaliar o comportamento do volume minuto, da fração expirada e do controle autonômico da FC durante a MASR em 16 homens jovens saudáveis, identificaram que não houve alterações da VFC durante a realização da manobra, e destacam que esta trata-se de uma técnica de mensuração segura para a avaliação da modulação parassimpática.

Nossos achados divergem da literatura contemporânea, uma vez que os amputados apresentaram uma marcada ativação simpática. Resultados semelhantes foram observados em estudos com outras populações como 
Doença Pulmonar Obstrutiva Crônica (DPOC) ${ }^{13,22,23}$, Insuficiência Cardíaca Congestiva (ICC) ${ }^{24}$ e sujeitos com asma ${ }^{25}$. Pacientes com DPOC, ICC e asma apresentam um incremento da atividade simpática e atenuação parassimpática durante a MARS e esse comportamento pode ser justificado devido às alterações de força muscular respiratória e comprometimento pulmonar, demonstrando maior 'turbulência' na função do SNA em comparação com a manobra ativa postural/simpática ${ }^{13,22-25}$. Curiosamente, ao avaliar o efeito da pressão positiva expiratória das vias aéreas (EPAP) com $5 \mathrm{cmH}_{2} \mathrm{O}$ sobre os índices de VFC em repouso e durante um teste de caminhada constante em pacientes com DPOC, Goulart et al. ${ }^{26}$ observaram melhora da modulação cardíaca e complexidade do SNA durante o repouso.

Este estudo apresentou algumas limitações que merecem ser mencionadas. Destacamos o pequeno $n$ amostral e a dificuldade em encontrar pacientes amputados por causa traumática. Devido aos critérios de exclusão da pesquisa nossa amostra não é totalmente representativa da população de amputados. Entretanto, a maioria de estudos prévios de análise da VFC obteve amostras relativamente semelhantes. Sabendo que há escassez de informações na literatura sobre modulação autonômica cardíaca em amputados, o presente estudo pode ter grande significado e relevância na reabilitação de amputados, uma vez que promovem uma melhor compreensão dos efeitos da amputação sobre o SNA, bem como, através do polar, um método não invasivo e de fácil aplicação, proporcionar uma avaliação desses pacientes em repouso e durante exercícios. Sugerimos um aumento do $\mathrm{n}$ amostral para estudos futuros, bem como a inserção de um grupo controle para comparação.

Por fim, nós concluímos que homens amputados unilaterais de membro inferior apresentam comportamento esperado durante a mudança ativa de postura, verificado através do aumento da ativação simpática. Entretanto, estes obtiveram respostas adversas durante a posição em supino e na MASR com atenuação vagal. Ademais, a maior modulação da atividade simpática nos sujeitos deste estudo esteve relacionada à causa traumática da amputação.

\section{REFERÊNCIAS}

1. Stefanic M, Presern-Strukelj M, Vidmar G, Kotnik V, Kopitar NA, Ihan A. Immunological status in patients with lower limb amputation due to peripheral arterial disease before and after comprehensive rehabilitation. Collegium Antropologicum. 2015;39(1):12530.

2. Vieira CFD, Lima MMO, Costa HS, Diniz KMA, Guia JPL, Alves FL et al. Correlation between indexes of autonomic maneuvers and heart rate variability in hemodialysis patients. Clinical Autonomic Research. 2016;26(3):181-8.

3. Peles E, Akselrod S, Goldstein DS, Nitzan H, Azaria $\mathrm{M}, \mathrm{Almog} \mathrm{S}$ et al. Insulin resistance and autonomic function in traumatic lower limb amputees. Clinical Autonomic Research. 1995; 5(5):279-88.

4. Knezevic A, Salamon T, Milankov M, Ninkovic S, Jeremic Knezevic M, Tomasevic Todorovic S. Assessment of quality of life in patients after lower limb amputation. Medicinski Pregled. 2015; 68(3):103-8.

5. Roque AL, Valenti VE, Massetti T, da Silva TD, Monteiro CBM, Oliveira FR et al. Chronic obstructive pulmonar disease and heart rate variability: a literature update. International Archives of Medicine. 2014;7(1): 43.

6. Metelka R. Heart rate variability - current diagnosis of the cardiac autonomic neuropathy. A Review. Biomedical Papers of the Medical Faculty of Palacky University in Olomouc. 2014; 158(3):327-38.

7. Monteze NM, Souza BB, Alves HJP, de Oliveira FL, de Oliveira JM, de Freitas SN et al. Heart rate variability in shift workers: responses to orthostatism and relationships with anthropometry, body composition, and blood pressure. Biomed Research International. 2015;2015:329057.

8. Vanderlei LCM, Pastre CM, Hoshi RA, Carvalho TD, Godoy MF. Basic notions of heart rate variability and its clinical applicability. Brazilian Journal of Cardiovascular Surgery. 2009; 24(2):205-17.

9. Cachadiña ES, García PG, da Luz SCT, Esteban RG, Pérez OB, Orellana JN et al. Heart rate variability and phantom pain in male amputees: application of linear and nonlinear methods. Journal Rehabilitation 
Research and Development. 2013;50(3):449-54.

10. Cooper KH. The aerobics way. Bantam Books, 1982.

11. Deé K, Lelovics Z. Correct determination of body mass index in people with lower limb amputation. Advanced Research in Scientific Areas. 2012;3(7):2133-37.

12. Osterkamp LK. Current perspective on assessment of human body proportions of relevance to amputees. Journal of the American Dietetic Association 1995; 95(2):215-18.

13. Reis MS, Arena R, Deus AP, Simões RP, Catai AM, Borghi-Silva A. Deep breathing heart rate variability is associated with respiratory muscle weakness in patients with chronic obstructive pumonary disease. Clinics 2010; 65(4):369-75.

14. Vaz MI, Roque V, Pimentel S, Rocha A, Duro H. Psychosocial characterization of a Portuguese lower limb amputee population. Acta Medica Portuguesa. 2012; 25(2):77-82.

15. Nunan D, Sandercock GRH, Brodie DA. Quantitative Systematic Review of Normal Values for Short-Term Heart Rate Variability in Healthy Adults. Pacing and Clinical Eletrophysiology. 2010;33(11):1407-17.

16. Jiang XL, Zhang ZG, Chen Y, Ye CP, Lei Y, Wu L et al. A blunted sympathetic and accentuated parasympathetic response to postural change in subjects with depressive disorders. Journal of Affective Disorders 2015;175:269-74.

17. Mahananto F, Tomohiko I, Murayama N. Potential force dynamics of heart rate variability reflect cardiac autonomic modulation with respect to posture, age, and breathing pattern. Computers in Biology and Medicine. 2015;64:197-207.

18. Loebenstein BG, Korn A, Waldhausl W. The role of adrenergic mechanisms in the blood pressure regulation of leg-amputees. Basic Research Cardiology 1981; 76(3):267-75.

19. Almeida CC. Estudo da variabilidade da frequência cardíaca e do nível funcional de amputados transfemorais (Programa de Pós-Graduação em Engenharia Biomédica) [dissertação]. Brasília: Universidade de Brasília - Faculdade UnB Gama; 2015. 101p.
20. Vuksanović V, Gal V. Nonlinear and chaos characteristics of heart period time series: healthy aging and postural change. Autonomic Neuroscience. 2005;121 (1-2):94-100.

21. Fenley A, Silva LDC, Reis HV, Sampaio LM, Borghi-Silva A, Reis MS. Cardiorespiratory adjustments during the accentuation of respiratory sinus arrhythmia: influence from time of maneuver on minute volume, fraction of expired $\mathrm{CO}_{2}$, and heart rate variability. Fisioterapia e Pesquisa. 2016;23(1):68-73.

22. Mazzuco A, Medeiros WM, Sperling MP, de Souza AS, Alencar MCN, Arbex FF et al. Relationship between linear and nonlinear dynamics of heart rate and impairment of lung function in COPD patients. International Journal of Chronic Obstructive Pulmonary Disease. 2015;10:1651-61.

23. Goulart C da L, Simon JC, Schneiders P de B, San Martin EA, Cabiddu R, Borghi-Silva A et al. Respiratory muscle strength effect on linear and nonlinear heart rate variability parameters in COPD patients. International Journal of Chronic Obstructive Pulmonary Disease. 2016;11:1671-1677.

24. Reis MS, Arena R, Archiza B, de Toledo CF, Catai AM, Borghi-Silva A. Deep breathing heart rate variability is associated with inspiratory muscle weakness in chronic heart failure. Physiotherapy Research International. 2014;19 1):16-24.

25. Garcia-Araújo AS, Di Lorenzo VA, Labadessa IG, Jürgensen SP, Di Thommazo-Luporini L, Garbim CL et al. Increased sympathetic modulation and decreased response of the heart rate variability in controlled asthma. Journal of Asthma. 2015;52(3):246-53.

26. Goulart CL, San Martin EA, Mansour KMK, Schneiders PB, Silva ALG. Influence of expiratory positive airway pressure on cardiac autonomic modulation at rest and in submaximal exercise in COPD patients. Brazilian Journal of Medical and Biological Research. 2018;51(6):e7180.

Recebido em: 18/09/2018

Aceito em: 28/12/2018 